\title{
Curcuma wenyujin Root Oil
}

National Cancer Institute

\section{Source}

National Cancer Institute. Curcuma wenyujin Root Oil. NCI Thesaurus. Code C107297.

The oil extracted from the root tuber of Curcuma wenyujin. 